\title{
Correlation of serum 25-hydroxy vitamin D with Leptin and visceral fat area in T2DM patients combined with osteoporosis.
}

\author{
Zhe Wang ${ }^{1}$, Yong-peng Wang ${ }^{2}$, Zai-rong Wei ${ }^{3}$, Li-ya Luo ${ }^{1}$, Yan Yang ${ }^{*}$, Yu-yu Zhu ${ }^{1}$, Qi Li ${ }^{1}$, Si-tong \\ Fan $^{1}$ \\ 1Department of Endocrinology, Affiliated Hospital of Zunyi Medical University, Guizhou Province, China \\ 2Department of Radiology, Affiliated Hospital of Zunyi Medical University, Guizhou Province, China
}

3Department of Burn and Plastic Surgery, Affiliated Hospital of Zunyi Medical University, Guizhou Province, China

\begin{abstract}
Purpose: To investigate the correlation of serum 25-hydroxy vitamin D [25(OH)D] with leptin (LEP) and visceral fat area (VFA) in type 2 diabetes mellitus (T2DM) patients with osteoporosis (OP).

Methods: A total of 135 T2DM patients with newly diagnosed admitted to the Department of Endocrinology, Affiliated Hospital of Zunyi Medical College. The patients were divided into three groups according to BMD status: (1) DMNB group, $n=45$; (2) DMLB group, $n=45$; and (3) DMOP group, $n=45$. The control group consisted of 50 age and gender matched subjects recruited from the Physical Examination Center (NC group). All patients were examined for general biochemical indicators and QCT was used to measure VFA. Electrochemiluminescence (ECL) and enzyme linked immunosorbent assay (ELISA) were used to evaluate the serum levels of 25 (OH)D and LEP, respectively.

Results: Compared with NC group, the levels of $25(\mathrm{OH}) \mathrm{D}, \mathrm{L1}-\mathrm{L} 4$ lumbar vertebrae and left femoral neck BMD in DMNB group, DMLB group and DMOP group were significantly lower $(P<0.05)$, the LEP and VFA levels were significantly increased $(P<0.05)$.

Conclusions: Our data showed a strong correlation between decreased 25 (OH)D and increased LEP serum levels in T2DM patients presenting with comorbid OP. This patient cohort also exhibited significantly higher VFA, which is symptomatic of imbalanced glucose and lipid metabolism.
\end{abstract}

Keywords: Type 2 diabetes, Osteoporosis, 25-hydroxyvitamin D, Leptin, Visceral fat area.

Accepted on July 04, 2019

\section{Introduction}

Type 2 diabetes mellitus (T2DM) and osteoporosis (OP) are metabolic diseases which, with rising living standards and aging populations, now commonly present in the same patients. Given their chronic nature, these disorders threaten to erase the gains to health and longevity that have accrued from medical advances during the past half century. Identification of risk factors that predispose for T2DM combined with OP is therefore important for early clinical control. The bone microenvironment and rate of bone formation in patients with diabetes and OP have undergone systemic changes due to the long-term effects of high insulin and hyperglycemia, which decreases bone formation by osteoblast (OB) and increases bone resorption by osteoclasts (OC), thus resulting in decreased BMD and subsequent OP [1]. Increased visceral fat area (VFA) leads to abnormal secretion of adipokines that adversely affect glucose, fat, and bone metabolism [2,3], as well as insulin sensitivity [4]. Adipokines are closely linked to T2DM but also affect the balance of bone metabolism through multiple pathways. Vitamin D is a fat-soluble vitamin which, in addition to its classical role of regulating the calcium and phosphorus metabolism, is closely linked to glucose and lipid metabolism [5]. Patients with T2DM have a higher prevalence of OP and low serum 25-hydroxy vitamin D [25 (OH)D] which affect the bone turnover by increasing bone resorption and impede bone formation in T2DM patients [6]. LEP is a small peptide hormone secreted by fat cells with bone being one of its target tissues [7]. Whereas LEP is a cytokine intrinsically associated with fat cells, serum $25(\mathrm{OH}) \mathrm{D}$ is representative of the level of vitamin $\mathrm{D}$ in the body and both molecules are closely related to bone metabolism [8,9]. Besides their roles in the metabolism of sugar, lipids and bone homeostasis, LEP and vitamin $\mathrm{D}$ have also been associated with the occurrence and development of T2DM, dyslipidemia and OP [5,10]. To date, there are no reported studies looking at the relationship between OP and LEP in T2DM patients. Therefore, in this study we investigated a cohort of patients presenting with both T2DM and OP to assess the correlation of serum levels of 25 $(\mathrm{OH}) \mathrm{D}$ with LEP and VFA. Our data showed a correlation of 
elevated LEP in T2DM patients with low BMD and may point to new ideas for the early prevention and treatment of T2DM and OP.

\section{Materials and Methods}

\section{Subjects}

A total of 135 T2DM patients with newly diagnosed were recruited for this study, between February 2017 and March 2018, having been diagnosed at the Department of Endocrinology, Affiliated Hospital of Zunyi Medical College. Each patient's BMD was assessed by Dual energy X-ray absorptiometry (DXA) and, referring to the Diagnosis and Treatment Criteria of Primary Osteoporosis issued in 2017 by the Osteoporosis and Bone Mineral Disease Branch of Chinese Medical Association, were allocated into one of three groups according to BMD: The normal bone mass group (DMNB, $\mathrm{n}=45$, age $60.26 \pm 7.12$ years old, 26 males and 19 females); the bone mass reduction group (DMLB, $n=45$, age $61.53 \pm$ 9.86 years old, 27 males and 18 females), and the osteoporosis group (DMOP, $\mathrm{n}=45$, age $61.94 \pm 9.23$ years old, 25 males and 20 females). Fifty healthy age and sex matched subjects were recruited from the Physical Examination Center and served as the normal control group (NC, age $60.06 \pm 6.96$ years old, 30 males and 20 females). Signed informed consent was given by every study subjects and the study was approved by the Ethics Committee of Zunyi Medical College.

\section{Inclusion criteria}

a) Diagnosed as T2DM according to the Diagnostic Criteria of Diabetes by 1999 WHO; b) postmenopausal females (menopause time $\geq 1$ year); c) male aged $\geq 60$ years; d. no history of smoking or long-term heavy alcohol consumption; d) Has not received any diabetes treatment including diet, drug intervention and exercise therapy.

\section{Exclusion criteria}

a) Individuals with severe organic diseases or acute and chronic complications of diabetes; b) with severe heart, liver, kidney, or other organ diseases; c) with rheumatoid arthritis, thyroid, bone tumours, pituitary, parathyroid, adrenal gland, gonad, or other diseases that may affect the bone metabolism; d) having taken drugs that may affect the bone metabolism 6 months before the examination, such as high-dose calcium, oestrogen, androgen, diphosphate, or glucocorticoids; e) having received hormone replacement therapy in the past 3 months; f) used contraceptive pills within 6 months; g) females in pregnancy, breast-feeding, or pregnancy preparation period; h) having taken oral or injected vitamin D within 8 months; i) with malignant tumours.

\section{Diagnostic criteria for osteoporosis}

The diagnostic criteria for osteoporosis as outlined in the Diagnosis and Treatment criteria of Primary Osteoporosis (2017). BMD measurements by DXA is the current general diagnostic indicator of osteoporosis: a BMD within 1 standard deviation (sd) of the peak BMD in healthy adults of the same sex and ethnicity is defined as normal; BMD reduced by 1 to $2.5 \mathrm{sd}$ is defined as low bone mass; whereas BMD reduced by more than $2.5 \mathrm{sd}$ is defined as osteoporosis; the degree of BMD reduction meeting the diagnostic criteria for osteoporosis, together with one or more brittle fractures, is defined as severe osteoporosis. The BMD is usually expressed as a T-Score, which is equal to (actual value-peak BMD in healthy adults of the same sex and ethnicity) / (sd of the peak BMD in healthy adults of the same sex and ethnicity). The diagnostic criterion for osteoporosis based on DXA-measured axial bone density (L1-4, femoral neck, or total hip), or distal 1/3 radial bone, is T-score $\leq 2.5$.

\section{Detection methods}

General information: The study subjects' gender, age, course of disease, body height, body weight, waist circumference, and hip circumference were collected and used to calculate the waist to hip ratio (WHR) and BMI.

Detection of biochemical indices: After an overnight fast, 5 $\mathrm{ml}$ of venous blood was collected from each study subject early the following morning. The blood samples were allowed to settle for $20 \mathrm{~min}$ at room temperature, followed by $10 \mathrm{~min}$ centrifugation at $3800 \mathrm{rpm}$ for biochemical testing (one sample) and further tests (storing a backup sample at -80). LEP serum levels were measured by ELISA; the level of $25(\mathrm{OH}) \mathrm{D}$ was measured by ECL using a Roche E160 kit (Roche Diagnostics $\mathrm{GmbH}$ ); the HbA1c was measured by HPLC using D-10 reagents (BioRad, USA); the levels of HDL-C, LDL-C, TG, and TC were tested using an Olympus automatic analyzer (AU2700); the Fasting plasma glucose (FPG) and postprandial blood glucose $(2 \mathrm{hPG})$ were tested using the hexose oxidase method; the levels of $\beta$-collagen degradation products $(\beta$ CTX), type I-collagen amino end lengthening peptide (PINP), and Fasting insulin (FINS) were assayed using the Roche E160 ECL kit; the HOMA-IR=FBG $\times$ FINS $/ 22.5$; the QUICKI=1/Log (I0)+Log (G0) [I0: fasting insulin content $(\mu \mathrm{U} / \mathrm{ml})$; $\mathrm{G} 0$ : fasting blood glucose concentration $(\mathrm{mg} / \mathrm{dl})]$.

Measurement of BMD: BMDs (unit: $\mathrm{g} / \mathrm{cm} 2$ ) of the orthotopical L1-4, proximal left femur (including the femoral neck), Ward's trigone, and total hip were measured using the Medix90 DMS (MEDILINK, France).

Assessing abdominal fat distribution: Quantitative CT was used for abdominal fat measurements using a GE Discovery CT750 HD. The scanning parameters were: $120 \mathrm{kV} \mathrm{150-400}$ $\mathrm{Ma}, 0.6 \mathrm{~S}$, scanning layer thickness $5 \mathrm{~mm}$, interlayer spacing 5 mm, pitch $0.984: 1$, matrix $512 \times 512$, and AsiR as SS30 slice $30 \%$ standard algorithm. The reconstructed layer thickness was $0.625 \mathrm{~mm}$, and the reconstructed layer spacing was $0.625 \mathrm{~mm}$. Each patient was placed in a supine position and scanned at the umbilical L2 3 in a breathless state. The reconstructed thinlayer data were transmitted to the GE AW4.5 workstation for analysis. The subcutaneous fat area of interest was drawn along the abdominal skin contour and the outer edge of abdominal wall muscles. The peripheral and retroperitoneal fat area of 
interest was drawn along the inner edge of abdominal wall muscles and the anterior spine. After having set up the fat density range, the measurements of all fat areas and percentages were completed on one computer by the same operator from the Department of Imaging.

Statistical methods: Statistical analysis was performed using the SPSS17.0 software. The measurement data were expressed as $(\bar{x} \pm \mathrm{sd})$. Analysis of variance was used for the intergroup comparison. The correlation among variables was analysed using the Pearson test. The multivariate logistic regression analysis was used to analyse the influencing factors, with $\mathrm{P}<0.05$ considered statistically significant.

\section{Results}

\section{Comparison of general information and clinical biochemical indices among groups}

General patient data and a comparison of biochemical indicators between the four groups are shown in Table 1 .
Of note was a significant decrease in BMD of L1-L4 and left femoral neck in the DMOP group when compared with the NC group $(\mathrm{P}<0.05)$. There was no significant difference Ward's trigone and total hip density across the four test groups $(\mathrm{P}>0.05)$ (Table 2).

VFA levels reached the highest values in the DMOP group at $136.62 \pm 28.64 \mathrm{~cm}^{2}$, compared to $90.46 \pm 17.32$ in $\mathrm{NC}, 95.87 \pm$ 20.36 in DMNB, and $130.89 \pm 22.79$ in DMLB $(\mathrm{P}<0.05)$ (Figure 1).

The level of $25(\mathrm{OH}) \mathrm{D}$ was significantly reduced in the DMOP group to $10.54 \pm 2.77 \mathrm{ng} / \mathrm{mL}$, compared to $28.16 \pm 4.17$ in the $\mathrm{NC}$ group, $21.12 \pm 3.38$ in the DMNB group, and $15.68 \pm 3.52$ in the DMLB group $(\mathrm{P}<0.05)$ (Figure 2).

LEP levels showed a 3-fold increase in DMOP $(11.26 \pm 3.27$ $\mathrm{ug} / \mathrm{L})$ group compared to $\mathrm{NC}$ group $(4.23 \pm 2.18)$, whereas there was a 1.5 -fold $(6.37 \pm 2.26)$ and $\sim 2$-fold $(11.26 \pm 3.27)$ increase, respectively, in the DMNB and DMLB groups $(\mathrm{P}<0.05)$ (Figure 3).

Table 1: Comparison of general information and biochemical indices among the four groups $\left(x^{-} \pm s d\right)$.

\begin{tabular}{|c|c|c|c|c|c|c|c|c|c|c|c|}
\hline Group & $N(M / F)$ & Age (years) & $\begin{array}{l}\text { Disease } \\
\text { duration } \\
\text { (years) }\end{array}$ & BMI $\left(\mathrm{Kg} / \mathbf{m}^{2}\right)$ & WHR & HbA1c (\%) & FPG (mmol/L) & & FINS (ulU/mL) & HOMA-IR & QUICKI \\
\hline NC & $50(30 / 20)$ & $60.06 \pm 6.96$ & - & $22.97 \pm 2.17$ & $0.71 \pm 0.04$ & $5.47 \pm 0.59$ & $6.18 \pm 0.54$ & & $15.58 \pm 6.74$ & $4.27 \pm 1.32$ & $0.50 \pm 0.04$ \\
\hline DMNB & $45(26 / 19)$ & $60.26 \pm 7.12$ & $7.34 \pm 4.52$ & $25.05 \pm 1.89^{\star}$ & $0.81 \pm 0.06^{*}$ & $7.56 \pm 0.48^{*}$ & $9.52 \pm 0.76^{*}$ & & $25.64 \pm 5.89^{\star}$ & $7.25 \pm 1.17^{*}$ & $0.41 \pm 0.02^{*}$ \\
\hline DMLB & $45(27 / 18)$ & $61.53 \pm 9.86$ & $7.98 \pm 4.78$ & $27.96 \pm 1.6^{\star} \#$ & $\begin{array}{l}0.98 \\
0.09^{\star} \#\end{array}$ & $8.97 \pm 0.67^{\star} \#$ & $11.19 \pm 0.57^{*}$ & & $26.07 \pm 5.26^{*}$ & $9.97 \pm 1.06^{\star} \#$ & $\begin{array}{l}0.34 \\
0.05^{\star} \#\end{array}$ \\
\hline DMOP & $45(25 / 20)$ & $61.94 \pm 9.23$ & $7.56 \pm 3.98$ & $\begin{array}{l}27.34 \\
1.90^{*} \#\end{array}$ & $\begin{array}{l}0.95 \\
0.07^{\star} \#\end{array}$ & $\begin{array}{l}10.18 \\
0.52^{*} \# \Delta\end{array}$ & $11.87 \pm 0.65^{*}$ & & $26.32 \pm 6.57^{*}$ & $\begin{array}{l}11.94 \\
1.83^{*} \# \Delta\end{array}$ & $\begin{array}{l}0.23 \\
0.06^{*} \# \Delta\end{array}$ \\
\hline Group & $\begin{array}{l}\text { TG } \\
\text { (mmol/L) }\end{array}$ & $\mathrm{TC}(\mathrm{mmol} / \mathrm{L})$ & $\begin{array}{l}\text { HDL-C } \\
\text { (mmol/L) }\end{array}$ & $\begin{array}{l}\text { LDL-C } \\
\text { (mmol/L) }\end{array}$ & $\begin{array}{l}\beta-C T X \\
(n g / m L)\end{array}$ & PINP (ng/mL) & VFA $\left(\mathrm{cm}^{2}\right)$ & & $\begin{array}{l}\text { 25(OH)D } \\
\text { (ng/mL) }\end{array}$ & Leptin (ug/L) & \\
\hline NC & $0.93 \pm 0.41$ & $4.27 \pm 0.37$ & $1.88 \pm 0.06$ & $2.09 \pm 0.37$ & $0.68 \pm 0.10$ & $51.89 \pm 12.12$ & $90.46 \pm 17.32$ & & $28.16 \pm 4.17$ & $4.23 \pm 2.18$ & \\
\hline DMNB & $2.74 \pm 0.26^{*}$ & $5.63 \pm 0.89^{*}$ & $0.94 \pm 0.05^{*}$ & $3.88 \pm 0.41^{*}$ & $1.34 \pm 0.12^{*}$ & $21.25 \pm 10.15^{\star}$ & $95.87 \pm 20.36$ & & $21.12 \pm 3.38^{*}$ & $6.37 \pm 2.26^{*}$ & \\
\hline DMLB & $2.96 \pm 0.35^{\star}$ & $6.41 \pm 0.91^{*}$ & $\begin{array}{l}0.73 \\
0.04^{\star} \#\end{array}$ & $3.93 \pm 0.32^{*}$ & $\begin{array}{l}1.89 \\
0.26^{\star} \#\end{array}$ & $15.63 \pm 7.01^{*} \#$ & $\begin{array}{l}130.89 \\
22.79^{*} \#\end{array}$ & \pm & $15.68 \pm 3.52^{*} \#$ & $8.79 \pm 2.37^{\star} \#$ & \\
\hline DMOP & $2.73 \pm 0.27^{*}$ & $6.90 \pm 0.54^{*}$ & $\begin{array}{l}0.61 \\
0.07^{\star} \# \Delta\end{array}$ & $4.17 \pm 0.40^{*}$ & $\begin{array}{l}2.14 \\
0.34^{\star} \# \Delta\end{array}$ & $5.12 \pm 2.15^{\star} \# \Delta$ & $\begin{array}{l}136.62 \\
28.64^{*} \#\end{array}$ & \pm & $\begin{array}{l}10.54 \\
2.77^{\star} \# \Delta\end{array}$ & $\begin{array}{l}11.26 \\
3.27^{*} \# \Delta\end{array}$ & \\
\hline
\end{tabular}

vs Group NC, *P0.05;vs Group DMNB, \#P0.05;vs Group DMLB, $\Delta \mathrm{P} 0.05$.

Table 2: Comparison of bone mineral density in four groups $\left(x^{-} \pm s d\right)$.

\begin{tabular}{|c|c|c|c|c|}
\hline Group & BMD of L1-L4 $\left(\mathrm{g} / \mathrm{cm}^{2}\right)$ & BMD of left femoral neck $\left(\mathrm{g} / \mathrm{cm}^{2}\right)$ & BMD of Ward's trigone $\left(\mathrm{g} / \mathrm{cm}^{2}\right)$ & BMD of total hip $\left(\mathrm{g} / \mathrm{cm}^{2}\right)$ \\
\hline NC & $1.24 \pm 0.12$ & $0.98 \pm 0.16$ & $0.70 \pm 0.18$ & $0.81 \pm 0.17$ \\
\hline DMNB & $1.12 \pm 0.11^{*}$ & $0.90 \pm 0.18^{*}$ & $0.69 \pm 0.10$ & $0.80 \pm 0.21$ \\
\hline DMLB & $0.88 \pm 0.08^{*} \#$ & $0.78 \pm 0.12^{* \#}$ & $0.67 \pm 0.08$ & $0.79 \pm 0.11$ \\
\hline DMOP & $0.64 \pm 0.06^{*} \# \Delta$ & $0.56 \pm 0.15^{*} \# \Delta$ & $0.66 \pm 0.12$ & $0.78 \pm 0.18$ \\
\hline
\end{tabular}

vs Group NC, "P0.05;vs Group DMNB, \#P0.05;vs Group DMLB, $\Delta \mathrm{P} 0.05$. 


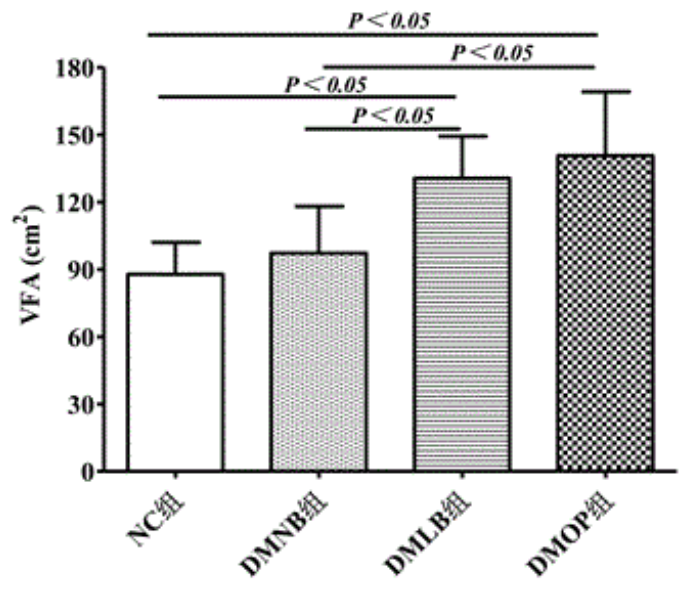

Figure 1: Comparison of VFA in the four test groups.

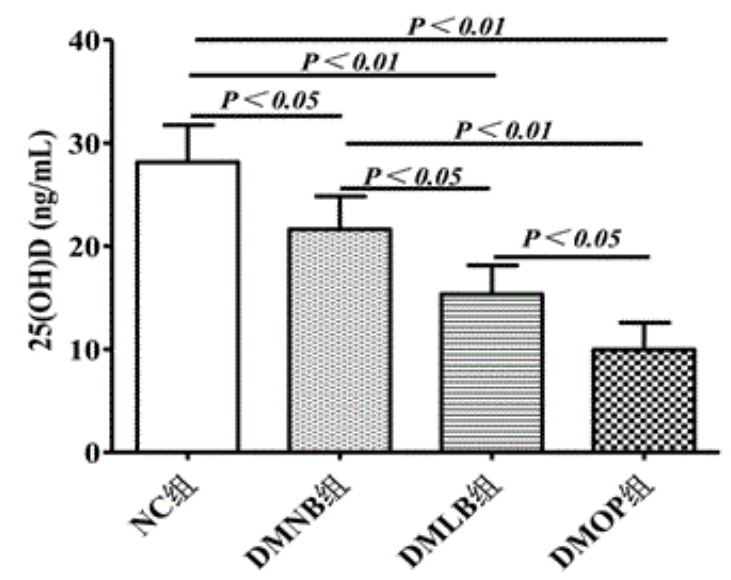

Figure 2: Comparison of serum 25 (OH) D levels between the four test groups.

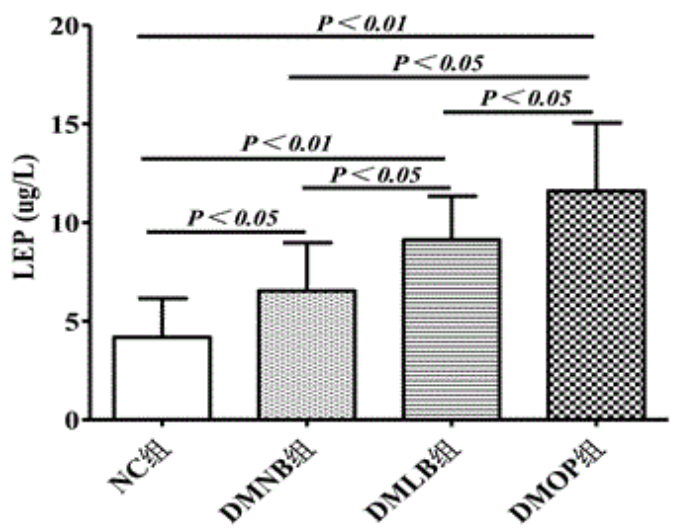

Figure 3: Comparison of serum Leptin levels between the four test groups.

\section{Pearson correlation analysis of serum 25(OH)D and clinical biochemical indices $(r)$}

When $25(\mathrm{OH}) \mathrm{D}$ was set as a dependent variable and each index as an independent variable, the correlation analysis showed that serum $25(\mathrm{OH}) \mathrm{D}$ was negatively correlated with LEP and VFA $(\mathrm{r}=-0.437$ and $-0.546, \mathrm{P}<0.05$, respectively) (Table 3).

Table 3: Correlation of 25(OH)D with general data and biochemical indexes ( $r$ ).

\begin{tabular}{lll}
\hline Items & \multicolumn{2}{|c|}{ 25(OH)D } \\
\cline { 2 - 3 } & $\mathbf{r}$ & $\mathbf{P}$ \\
\hline LEP $(\mathrm{ug} / \mathrm{L})$ & -0.437 & 0.03 \\
\hline VFA $\left(\mathrm{cm}^{2}\right)$ & -0.546 & 0.05 \\
\hline
\end{tabular}

\section{Logistic regression analysis of risk factors for T2DM combined with $\mathrm{OP}$}

The multivariate logistic regression analysis was performed, setting the combination of T2DM and OP or not as a dependent variable and the 25(OH)D, LEP and VFA as an independent variable, targeting the four groups. This analysis showed that VFA was an independent risk factor for the combination of T2DM and OP, whereas 25(OH)D and LEP were protective factors (Table 4).

Table 4: Logistic regression analysis of risk factors for T2DM patients with $O P$.

\begin{tabular}{llllll}
\hline Variable & $\boldsymbol{\beta}$ & $\mathrm{SE}$ & Waldc2 & $\mathbf{P}$ & OR(95\%Cl) \\
\hline 25(OH)D $(\mathrm{ng} / \mathrm{mL})$ & -1.014 & 0.783 & 4.634 & 0.032 & $0.498(0.184 \sim 0.658)$ \\
\hline $\mathrm{LEP}(\mathrm{ug} / \mathrm{L})$ & -1.257 & 0.892 & 4.858 & 0.012 & $0.234(0.054 \sim 0.513)$ \\
\hline $\operatorname{VFA}\left(\mathrm{cm}^{2}\right)$ & 0.065 & 0.638 & 3.125 & 0.002 & $1.614(1.269 \sim 2.584)$ \\
\hline
\end{tabular}

\section{Discussion}

Asia has emerged as a major area in the epidemic spread of T2DM [11] with China having seen a $>300 \%$ increase of T2DM cases since 2000 [12]. Obesity in the form of visceral fat deposition is strongly associated with an increased risk of metabolic syndrome, which encompasses, elevated serum lipids, high blood pressure, atherosclerosis, IR and T2DM $[13,14]$. Epidemiological studies show that T2DM patients with comorbid OP have a higher fracture risk, compared to non-diabetic OP patients, despite having higher BMD levels, an indication that links T2DM to abnormal bone metabolism [15]. There is now ample evidence to suggest that vitamin D has a protective effect on the pancreatic islets [16] and under physiological conditions, the secretion of glucose-stimulated insulin and the maintenance of normal glucose tolerance depend on vitamin D [17]. Vitamin D deficiency is, therefore, an important trigger for the occurrence and development of T2DM [18] with 25(OH)D the most stable content in serum used to assess vitamin D status in human subjects [19]. 
Vitamin D predominantly originates from UVB-induced skin synthesis and is therefore considered a hormone rather than a vitamin. Vitamin D has multiple physiological roles and its deficiency $(<20 \mathrm{ng} / \mathrm{mL})$ has multiple implications for human health, not least in bone homeostasis, by maintaining normal blood calcium and phosphorus levels and promoting normal bone mineralization. Vitamin $\mathrm{D}$ deficiency is a reliable indicator for OP and increased fracture risk and is an independent risk factor for diabetic osteoporosis [20]. There are indications that the expression of genes such as CYP2J2 and CYP27B1, which encode enzymes responsible for vitamin $\mathrm{D}$ synthesis, are reduced in subcutaneous tissues in obese individuals and may, therefore, result in vitamin D deficiency [21]. Over the past few decades, there has been the realization that vitamin $\mathrm{D}$ not only affects bone metabolism but also interacts with many other tissues and affects several metabolic pathways including the fat cell metabolism [22]. An increase in body fat is closely related to a decrease of $25(\mathrm{OH}) \mathrm{D}$ in circulation, and this is particularly the case in T2DM patients with OP.

Excessive visceral fat deposition greatly influences the BMD, whereas SFA has no effect on the BMD. One study found that T2DM patients with high degree of obesity and visceral fat deposition had a decreased risk of osteopenia when insulin sensitivity, hypoglycemic treatment measures, and blood glucose were appropriately controlled [3]. However, research findings into obesity and BMD are often contradictory. For example, a study involving a cohort of 320 Chinese women performed CT scans targeting the SFA and VFA volumes and measured the BMD of L2-L4. The VFA volume was negatively correlated with the BMD in the $<55$-year-old group but showed no correlation with the BMD in the $>55$-year-old group. However, the SFA volume had no correlation with the BMD, regardless of age [23]. A cross-sectional study of 340 obese women in Italy, found that trunk fat negatively correlated with the BMD independent of vitamin D levels [24]. A large-sample study encompassing both Chinese and Caucasian subjects found no protective effects on bone mass deriving from body fat mass in obese individuals [25]. A report by Zhang et al. found a strong negative correlation between visceral fat deposits and the bone mass [26].

The results in the present study are consistent with those reported by Zhang et al., in that serum 25(OH)D levels gradually decreased commensurate with decreased bone mass (DMOP $<$ DMLB $<$ DMNB $<$ NC), and that the LEP and VFA gradually increased (DMOP $>$ DMLB $>$ DMNB $>N C$ ) Therefore, we hypothesize that lower serum $25(\mathrm{OH}) \mathrm{D}$ levels and higher LEP levels may result in abnormal lipid metabolism and ectopic fat deposition and lead not only to increased VFA-and a higher propensity for T2DM-but also to the occurrence and development of OP. The ectopic deposition of fat is not limited to the abdominal cavity but also occurs in the bone marrow, and this may be the key to the increase of VFA, as well as the occurrence of T2DM combined with OP in the same patient. However, the molecular mechanisms governing this phenomenon still requires further research.
LEP is a protein product encoded by the OB gene and synthesized by the adipocytes. It regulates blood sugar by inhibiting insulin secretion, and is closely related to obesity, dyslipidemia, insulin resistance and associated conditions [27]. In the present study, we found a strong association between high serum levels of LEP and T2DM. We hypothesize that LEP affects the severity of T2DM via the following mechanism. In this scenario LEP triggers the JAK2/SAT3/STAT5b signalling pathway to induce the signal transducer and activator of transcription 3 (STAT3) expression, which inhibits insulin synthesis. At the same time, the high LEP levels exacerbates insulin resistance, and the combination of lower insulin expression and insulin resistance leads to T2DM [28,29]. LEP is known to be associated with bone metabolism, and elevated LEP levels in obese patients may be a mechanism responsible for the increased bone mass often seen in these individuals [30,31]. We study found a positive correlation between LEP and BMD values which supports the notion of LEP as a positive regulator of bone mineral density [32]. However, the relationship between LEP and BMD has by no means been settled, with studies both in China and abroad reporting seemingly contradictory results. For example, a Turkish study of postmenopausal diabetic women found no relationship between low BMD and LEP, except for a small region in the femoral head known as the Ward triangle and which showed a negative correlation between BMD and LEP [33]. Other studies of obese postmenopausal women have found reduced oestrogen secretion which, when coupled with significantly increased visceral fat accumulation, was associated with the synthesis and secretion of LEP, which then regulated bone metabolism via the OPG/RANKL/RANK system [34]. In the present study, we found that plasma $25(\mathrm{OH}) \mathrm{D}$ levels were negatively correlated with LEP expression, and that bone mass decreased commensurate with a decrease in $25(\mathrm{OH}) \mathrm{D}$, whereas LEP levels increased. We therefore hypothesize that increased VFA, dyslipidemia and high blood glucose leads to elevated LEP and 25(OH)D levels and that elevated LEP and 25(OH)D levels may in turn promote increased VFA and sugar, lipid metabolic disorder. The increased VFA and disorders of sugar and lipid metabolism is likely to aggravate the $25(\mathrm{OH}) \mathrm{D}$ deficiency, setting up a negative feedback loop. Actively supplementing vitamin D may break this vicious cycle, but the specific mechanism still needs further studies and exploration.

\section{Conclusion}

In summary, serum $25(\mathrm{OH}) \mathrm{D}$ decreases with the decrease in bone mass in T2DM patients. Vitamin D deficiency was most pronounced in T2DM patients with OP, and the inverse relationship between $25(\mathrm{OH}) \mathrm{D}$ and LEP may affect lipid metabolism, thus increasing VFA and lead to the occurrence and development of T2DM and OP. Therefore, vitamin D supplementation, and lowering LEP by nutritional means will lower the body fat percentage and also have positive impact for the prevention and treatment of T2DM and OP. 


\section{Acknowledgements}

National Natural Science Foundation of China (81460168), Zunyi City Joint Fund \{Number: Zunshi Keheshe [2018] 63\}, Zunyi medical college after planted cultivation and innovation to explore the special allowance\{number: Guizhou branch platform for talents [2017] 5733-043\}.

\section{References}

1. Wongdee $\mathrm{K}$, Charoenphandhu $\mathrm{N}$. Update on type 2 diabetes-related osteoporosis. World J Diabetes 2015; 6: 673-678.

2. Yamamoto M. Diabetes mellitus and osteoporosis. Predictive candidate markers for fracture risk in diabetic patients. Clin Calcium 2012; 22: 1359-1365.

3. Walsh JS, Vilaca T. Obesity, Type 2 Diabetes and Bone in Adults. Calcif Tissue Int 2017; 100: 528-535.

4. Kusminski CM, Bickel PE, Scherer PE. Targeting adipose tissue in the treatment of obesity-associated diabetes. Nat Rev Drug Discov 2016; 15: 639-60.

5. Raska I, Raskova M, Zikan V, Skrha J. High Prevalence of Hypovitaminosis D in Postmenopausal Women with Type 2 Diabetes Mellitus. Prague Medical Report 2016; 117: 5-17.

6. Perez-Diaz I, Sebastian-Barajas G, Hernandez-Flores ZG, Rivera-Moscoso R, Osorio-Landa HK, Flores-Rebollar A. The impact of vitamin D levels on glycemic control and bone mineral density in postmenopausal women with type 2 diabetes. J Endocrinol Invest 2015; 38: 1365-1372.

7. Zhang Y, Proenca R, Maffei M, Barone M, Leopold L, Friedman JM. Positional cloning of the mouse obese gene and its human homologue. Nature 1994; 372: 425-432.

8. Chen XX, Yang T. Roles of leptin in bone metabolism and bone diseases. J Bone Miner Metab 2015; 33: 474-485.

9. Nemeth MV, Wilkens MR, Liesegang A. Vitamin D status in growing dairy goats and sheep: Influence of ultraviolet B radiation on bone metabolism and calcium homeostasis. Journal of Dairy Science 2017; 100: 8072-8086.

10. Vasilkova O, Mokhort T, Sharshakova T, Hayashida N, Takamura N. Leptin is an independent determinant of bone mineral density in men with type 2 diabetes mellitus. Acta Diabetologica 2011; 48: 291-295.

11. Zheng Y, Ley SH, Hu FB. Global aetiology and epidemiology of type 2 diabetes mellitus and its complications. Nat Rev Endocrinol 2018; 14: 88-98.

12. Yuan H, Li X, Wan G, Sun L, Zhu X, Che F, Yang Z. Type 2 diabetes epidemic in East Asia: a 35-year systematic trend analysis. Oncotarget 2018; 9: 6718-6727.

13. Kurozumi A, Okada Y, Arao T, Tanaka Y. Excess Visceral Adipose Tissue Worsens the Vascular Endothelial Function in Patients with Type 2 Diabetes Mellitus. Intern Med 2016; 55: 3091-5.

14. Wanderley-Rocha DR, Jorge AR, Braulio VB, Arbex AK, Marcadenti A. Visceral Adiposity Measurements, Metabolic and Inflammatory Profile in Obese Patients with and Without Type 2 Diabetes Mellitus: A Crosssectional Analysis. Curr Diabetes Rev 2017; 13: 11-18.
15. Goldshtein I, Nguyen AM, dePapp AE, Ish-Shalom S, Chandler JM, Chodick G, Shalev V. Epidemiology and correlates of osteoporotic fractures among type 2 diabetic patients. Arch Osteoporos 2018; 13: 15.

16. Jung CH, Kim KJ, Kim BY, Kim CH, Kang SK, Mok JO. Relationship between vitamin D status and vascular complications in patients with type 2 diabetes mellitus. Nutr Res 2016; 36: 117-124.

17. Palomer X, Gonzalez-Clemente JM, Blanco-Vaca F, Mauricio D. Role of vitamin D in the pathogenesis of type 2 diabetes mellitus. Diabetes Obes Metab 2008; 10: 185-197.

18. Mohamad MI, El-Sherbeny EE, Bekhet MM. The Effect of Vitamin D Supplementation on Glycemic Control and Lipid Profile in Patients with Type 2 Diabetes Mellitus. J Am Coll Nutr 2016; 35: 399-404.

19. Herrmann M, Sullivan DR, Veillard AS, McCorquodale T, Straub IR, Scott R, Laakso M, Topliss D, Jenkins AJ, Blankenberg S, Burton A, Keech AC. Serum 25hydroxyvitamin D: a predictor of macrovascular and microvascular complications in patients with type 2 diabetes. Diabetes Care 2015; 38: 521-528.

20. Brincat M, Gambin J, Brincat M, Calleja-Agius J. The role of vitamin D in osteoporosis. Maturitas 2015; 80: 329-332.

21. Pelczynska M, Grzelak T, Walczak M, Czyzewska K. Hypovitaminosis D and adipose tissue - cause and effect relationships in obesity. Ann Agric Environ Med 2016; 23: 403-409.

22. Chang E, Kim Y. Vitamin D Insufficiency Exacerbates Adipose Tissue Macrophage Infiltration and Decreases AMPK/SIRT1 Activity in Obese Rats. Nutrients 2017; 9-4.

23. Wang L, Wang W, Xu L, Cheng X, Ma Y, Liu D, Guo Z, Su Y, Wang Q. Relation of visceral and subcutaneous adipose tissue to bone mineral density in Chinese women. Int J Endocrinol 2013; 2013: 378632.

24. Greco EA, Francomano D, Fornari R, Marocco C, Lubrano C, Papa V, Wannenes F, Luigi LD, Donini LM, Lenzi A, Aversa A, Migliaccio S. Negative association between trunk fat, insulin resistance and skeleton in obese women. World J Diabetes 2013; 4: 31-39.

25. Zhao LJ, Liu YJ, Liu PY, Hamilton J, Recker RR, Deng HW. Relationship of obesity with osteoporosis. J Clin Endocrinol Metab 2007; 92: 1640-1646.

26. Zhang P, Peterson M, Su GL, Wang SC. Visceral adiposity is negatively associated with bone density and muscle attenuation. Am J Clin Nutr 2015; 101: 337-343.

27. Rehman K, Akash MSH, Alina Z. Leptin: A new therapeutic target for treatment of diabetes mellitus. J Cell Biochem 2018; 119: 5016-5027.

28. Moradi S, Mirzaei K, Abdurahman AA, Keshavarz SA. Adipokines may mediate the relationship between resting metabolic rates and bone mineral densities in obese women Osteoporos Int 2017; 28: 1619-1629.

29. Park HK, Ahima RS. Physiology of leptin: energy homeostasis, neuroendocrine function and metabolism. Metabolism 2015; 64: 24-34. 
Correlation of serum 25-hydroxy vitamin D with Leptin and visceral fat area in T2DM patients combined with osteoporosis

30. Martin A, David V, Malaval L, Lafage-Proust MH, Vico L, Thomas T. Opposite effects of leptin on bone metabolism: a dose-dependent balance related to energy intake and insulin-like growth factor-I pathway. Endocrinology 2007; 148: 3419-3425.

31. Kalra SP, Dube MG, Iwaniec UT. Leptin increases osteoblast-specific osteocalcin release through a hypothalamic relay. Peptides 2009; 30: 967-973.

32. Vondracek SF, Voelkel NF, McDermott MT, Valdez C. The relationship between adipokines, body composition, and bone density in men with chronic obstructive pulmonary disease. Int J Chron Obstruct Pulmon Dis 2009; 4: 267-277.

33. Koroglu BK, Kiris F, Ersoy IH, Sutcu R, Yildiz M, Aksu O, Ermis F, Ersoy S, Tamer MN. Relation of leptin, adiponectin and insulin resistance to bone mineral density in type 2 diabetic postmenopausal women. Endokrynologia Polska 2011; 62: 429-435.

34. Legiran S, Brandi ML. Bone mass regulation of leptin and postmenopausal osteoporosis with obesity. Clinical Cases in Mineral and Bone Metabolism. 2012; 9: 145.

\section{*Correspondence to}

Yan Yang

Department of Endocrinology

Affiliated Hospital of Zunyi Medical University

Guizhou Province

China 